\title{
Extension of functions with small oscillation
}

\author{
by \\ Denny H. Leung and Wee-Kee Tang (Singapore)
}

\begin{abstract}
A classical theorem of Kuratowski says that every Baire one function on a $G_{\delta}$ subspace of a Polish (= separable completely metrizable) space $X$ can be extended to a Baire one function on $X$. Kechris and Louveau introduced a finer gradation of Baire one functions into small Baire classes. A Baire one function $f$ is assigned into a class in this hierarchy depending on its oscillation index $\beta(f)$. We prove a refinement of Kuratowski's theorem: if $Y$ is a subspace of a metric space $X$ and $f$ is a real-valued function on $Y$ such that $\beta_{Y}(f)<\omega^{\alpha}, \alpha<\omega_{1}$, then $f$ has an extension $F$ to $X$ so that $\beta_{X}(F) \leq \omega^{\alpha}$. We also show that if $f$ is a continuous real-valued function on $Y$, then $f$ has an extension $F$ to $X$ so that $\beta_{X}(F) \leq 3$. An example is constructed to show that this result is optimal.
\end{abstract}

Let $X$ be a topological space. A real-valued function on $X$ belongs to Baire class one if it is the pointwise limit of a sequence of continuous functions. If $X$ is a Polish (= separable completely metrizable) space, then a classical theorem of Kuratowski [7] states that every Baire one function on a $G_{\delta}$ subspace of $X$ can be extended to a Baire one function on $X$. In [6], Kechris and Louveau introduced a finer gradation of Baire one functions into small Baire classes using the oscillation index $\beta$, whose definition we now recall.

Let $X$ be a topological space and let $\mathcal{C}$ denote the collection of all closed subsets of $X$. A derivation on $\mathcal{C}$ is a map $\mathcal{D}: \mathcal{C} \rightarrow \mathcal{C}$ such that $\mathcal{D}(P) \subseteq P$ for all $P \in \mathcal{C}$. The oscillation index $\beta$ is associated with a family of derivations. Let $\varepsilon>0$ and a function $f: X \rightarrow \mathbb{R}$ be given. For any $P \in \mathcal{C}$, let $\mathcal{D}^{0}(f, \varepsilon, P)=P$ and $\mathcal{D}^{1}(f, \varepsilon, P)$ be the set of all $x \in P$ such that for any neighborhood $U$ of $x$, there exist $x_{1}, x_{2} \in P \cap U$ such that $\left|f\left(x_{1}\right)-f\left(x_{2}\right)\right| \geq \varepsilon$. The derivation $\mathcal{D}^{1}(f, \varepsilon, \cdot)$ may be iterated in the usual manner. For all $\alpha<\omega_{1}$, let

$$
\mathcal{D}^{\alpha+1}(f, \varepsilon, P)=\mathcal{D}^{1}\left(f, \varepsilon, \mathcal{D}^{\alpha}(f, \varepsilon, P)\right) .
$$

2000 Mathematics Subject Classification: Primary 26A21; Secondary 03E15, 54C30.

Key words and phrases: Baire-1 functions, oscillation index, extension of functions. 
If $\alpha$ is a countable limit ordinal, set

$$
\mathcal{D}^{\alpha}(f, \varepsilon, P)=\bigcap_{\gamma<\alpha} \mathcal{D}^{\gamma}(f, \varepsilon, P) .
$$

If $\mathcal{D}^{\alpha}(f, \varepsilon, P) \neq \emptyset$ for all $\alpha<\omega_{1}$, let $\beta_{X}(f, \varepsilon)=\omega_{1}$. Otherwise, let $\beta_{X}(f, \varepsilon)$ be the smallest countable ordinal $\alpha$ such that $\mathcal{D}^{\alpha}(f, \varepsilon, P)=\emptyset$. The oscillation index of $f$ is $\beta_{X}(f)=\sup _{\varepsilon>0} \beta_{X}(f, \varepsilon)$.

The main result of $\S 1$ is that if $Y$ is a subspace of a metric space $X$ and $f: Y \rightarrow \mathbb{R}$ satisfies $\beta_{Y}(f)<\omega^{\alpha}$ for some $\alpha<\omega_{1}$, then $f$ can be extended to a function $F$ on $X$ with $\beta_{X}(F) \leq \omega^{\alpha}$. It follows readily from the Baire characterization theorem $[2,10.15]$ that a real-valued function on a Polish space is Baire one if and only if its oscillation index is countable (see, e.g., [6]). Also, a theorem of Aleksandrov says that a $G_{\delta}$ subspace of a Polish space is Polish $[2,10.18]$. Hence our result refines Kuratowski's theorem in terms of the oscillation index. Let us mention that if $X$ is a metric space, then every real-valued function with countable oscillation index on a closed subspace of $X$ may be extended to $X$ with preservation of the index [8, Theorem 3.6]. (Note that the proof of [8, Theorem 3.6] does not require the compactness of the ambient space.) More recent results on the extension of Baire one functions on general topological spaces are found in [5].

It is well known that if a function is continuous on a closed subspace of a metric space, then there exists a continuous extension to the whole space. $\S 2$ is devoted to the study of extensions of continuous functions from an arbitrary subspace of a metric space. It is shown that if $f$ is a continuous function on a subspace $Y$ of a metric space $X$, then $f$ has an extension $F$ to $X$ with $\beta_{X}(F) \leq 3$. An example is given to show that the result is optimal. The criteria for continuous extension from dense subspaces were studied by several authors (see, e.g., [1], [4]).

1. Functions of small oscillation. Given a real-valued function defined on a set $S$, let $\|f\|_{S}=\sup _{s \in S}|f(s)|$. Since we do not assume that the function $f$ is bounded, $\|f\|_{S}$ may take the value $+\infty$. For any topological space $X$, the support supp $f$ of a function $f: X \rightarrow \mathbb{R}$ is the closed set $\overline{\{x \in X: f(x) \neq 0\}}$. A family $\left\{\varphi_{\alpha}: \alpha \in \mathcal{A}\right\}$ of nonnegative, continuous real-valued functions on $X$ is called a partition of unity on $X$ if

(1) the supports of the $\varphi_{\alpha}$ 's form a locally finite closed covering of $X$,

(2) $\sum_{\alpha \in \mathcal{A}} \varphi_{\alpha}(x)=1$ for all $x \in X$.

If $\left\{U_{\beta}: \beta \in \mathcal{B}\right\}$ is an open covering of $X$, we say that a partition of unity $\left\{\varphi_{\beta}: \beta \in \mathcal{B}\right\}$ on $X$ is subordinate to $\left\{U_{\beta}: \beta \in \mathcal{B}\right\}$ if the support of each $\varphi_{\beta}$ lies in the corresponding $U_{\beta}$. It is well known that if $X$ is paracompact (in particular, if $X$ is a metric space [3, Theorem IX.5.3]), then for each open 
covering $\left\{U_{\beta}: \beta \in \mathcal{B}\right\}$ of $X$ there is a partition of unity on $X$ subordinate to $\left\{U_{\beta}: \beta \in \mathcal{B}\right\}$ (see, for example, [3, Theorem VIII.4.2]).

Proposition 1. Let $X$ be a metric space and $Y$ be a subspace of $X$. Suppose that $f: Y \rightarrow \mathbb{R}$ is a function such that $\beta_{Y}(f, \varepsilon) \leq \alpha$ for some $\varepsilon>0$, $\alpha<\omega_{1}$. Then there exists a function $\tilde{f}: X \rightarrow \mathbb{R}$ such that $\beta_{X}(\tilde{f}) \leq \alpha+1$, $\|\widetilde{f}\|_{X} \leq\|f\|_{Y}$ and $\|f-\widetilde{f}\|_{Y} \leq \varepsilon$.

In the following, denote $\mathcal{D}^{\beta}(f, \varepsilon, Y)$ by $Y^{\beta}$ for all $\beta<\omega_{1}$. Proposition 1 is proved by working on each of the pieces $Y^{\beta} \backslash Y^{\beta+1}, \beta<\alpha$, and gluing together the results.

Lemma 2. For all $0 \leq \beta<\alpha$, there exist an open set $Z_{\beta}$ in $X$ such that $Y^{\beta} \backslash Y^{\beta+1} \subseteq Z_{\beta} \subseteq\left(Y^{\beta+1}\right)^{\mathrm{c}}$, and a continuous function $f_{\beta}: Z_{\beta} \rightarrow \mathbb{R}$ such that $\left\|f-f_{\beta}\right\|_{Y^{\beta} \backslash Y^{\beta+1}} \leq \varepsilon$ and $\left\|f_{\beta}\right\|_{Z_{\beta}} \leq\|f\|_{Y}$.

Proof. If $0 \leq \beta<\alpha$ and $y \in Y^{\beta} \backslash Y^{\beta+1}$, there exists a set $U_{y}$ that is an open neighborhood of $y$ in $X$ so that $U_{y}$ is disjoint from $Y^{\beta+1}$ and that $f\left(U_{y} \cap Y^{\beta}\right) \subseteq(f(y)-\varepsilon, f(y)+\varepsilon)$. Let

$$
Z_{\beta}=\bigcup_{y \in Y^{\beta} \backslash Y^{\beta+1}} U_{y}
$$

Each $Z_{\beta}$ is open in $X$. Clearly, $Y^{\beta} \backslash Y^{\beta+1} \subseteq Z_{\beta} \subseteq\left(Y^{\beta+1}\right)^{\text {c }}$. There exists a partition of unity $\left(\varphi_{y}\right)_{y \in Y^{\beta} \backslash Y^{\beta+1}}$ on $Z_{\beta}$ subordinate to the open covering $\mathcal{U}=\left\{U_{y}: y \in Y^{\beta} \backslash Y^{\beta+1}\right\}$. Define $f_{\beta}: Z_{\beta} \rightarrow \mathbb{R}$ by

$$
f_{\beta}(z)=\sum_{y \in Y^{\beta} \backslash Y^{\beta+1}} f(y) \varphi_{y}(z) .
$$

Then $f_{\beta}$ is well defined, continuous and $\left\|f_{\beta}\right\|_{Z_{\beta}} \leq\|f\|_{Y}$. If $x \in Y^{\beta} \backslash Y^{\beta+1}$, set $V_{x}=\left\{y \in Y^{\beta} \backslash Y^{\beta+1}: \varphi_{y}(x) \neq 0\right\}$. Then $\sum_{y \in V_{x}} \varphi_{y}(x)=1$. If $y \in V_{x}$, then $x \in U_{y}$; thus $|f(x)-f(y)|<\varepsilon$. Hence

$$
\left|f(x)-f_{\beta}(x)\right|=\left|\sum_{y \in V_{x}}(f(x)-f(y)) \varphi_{y}(x)\right| \leq \sum_{y \in V_{x}}|f(x)-f(y)| \varphi_{y}(x) \leq \varepsilon .
$$

Therefore, $\left\|f-f_{\beta}\right\|_{Y^{\beta} \backslash Y^{\beta+1}} \leq \varepsilon$, as required.

Proof of Proposition 1. Define a function $\tilde{f}: X \rightarrow \mathbb{R}$ by

$$
\tilde{f}(x)= \begin{cases}f_{\beta}(x) & \text { if } x \in Z_{\beta} \backslash \bigcup_{\gamma<\beta} Z_{\gamma}, \beta<\alpha, \\ 0 & \text { if } x \notin \bigcup_{\gamma<\alpha} Z_{\gamma} .\end{cases}
$$

Clearly, $\|\widetilde{f}\|_{X}=\sup _{\beta<\alpha}\left\|f_{\beta}\right\|_{Z_{\beta}} \leq\|f\|_{Y}$. If $x \in Y$, then $x \in Y^{\beta} \backslash Y^{\beta+1}$ for some $\beta<\alpha$. In particular, $x \in Z_{\beta} \backslash \bigcup_{\gamma<\beta} Z_{\gamma}$. Hence $|f(x)-\widetilde{f}(x)|=$ $\left|f(x)-f_{\beta}(x)\right| \leq\left\|f-f_{\beta}\right\|_{Y^{\beta} \backslash Y^{\beta+1}} \leq \varepsilon$ according to Lemma 2 . Since this is true for all $x \in Y$, we have $\|f-\widetilde{f}\|_{Y} \leq \varepsilon$. 
It remains to show that $\beta_{X}(\widetilde{f}) \leq \alpha+1$. To this end, we claim that $\mathcal{D}^{\beta}(\tilde{f}, \delta, X) \cap Z_{\gamma}=\emptyset$ for all $\delta>0, \gamma<\beta \leq \alpha$. We prove the claim by induction. Let $\delta>0$. Since $f_{0}$ is continuous on the open set $Z_{0}$, we have $\mathcal{D}^{1}(\widetilde{f}, \delta, X) \cap Z_{0}=\emptyset$. Suppose that the claim holds for all ordinals less than $\beta$. By the inductive hypothesis, $\mathcal{D}^{\xi}(\widetilde{f}, \delta, X) \cap \bigcup_{\gamma<\xi} Z_{\gamma}=\emptyset$ for all $\xi<\beta$. Therefore,

$$
\mathcal{D}^{\xi}(\tilde{f}, \delta, X) \cap\left[Z_{\xi} \backslash \bigcup_{\gamma<\xi} Z_{\gamma}\right]=\mathcal{D}^{\xi}(\tilde{f}, \delta, X) \cap Z_{\xi} .
$$

Now $\tilde{f}=f_{\xi}$ is continuous on this set, which is open in $\mathcal{D}^{\xi}(\widetilde{f}, \delta, X)$. Therefore $\mathcal{D}^{\xi+1}(\widetilde{f}, \delta, X) \cap Z_{\xi}=\emptyset$. Also, since $\mathcal{D}^{\beta}(\widetilde{f}, \delta, X) \subseteq \mathcal{D}^{\gamma+1}(\widetilde{f}, \delta, X)$ for all $\gamma<\beta$,

$$
\mathcal{D}^{\beta}(\widetilde{f}, \delta, X) \cap Z_{\gamma}=\emptyset
$$

for all $\gamma<\beta$. This proves the claim. It follows from the claim that

$$
\mathcal{D}^{\alpha}(\tilde{f}, \delta, X) \subseteq\left(\bigcup_{\gamma<\alpha} Z_{\gamma}\right)^{\mathrm{c}}
$$

for any $\delta>0$. Since $\widetilde{f}=0$ on the latter set, $\mathcal{D}^{\alpha+1}(\widetilde{f}, \delta, X)=\emptyset$.

In order to iterate Proposition 1 to obtain an extension of $f$, we need the following result.

Proposition 3. Let $Y$ be a subspace of a metric space $X$. If $\beta_{Y}(f)<\omega^{\xi}$ and $\beta_{Y}(g)<\omega^{\xi}$, then $\beta_{Y}(f+g)<\omega^{\xi}$.

Proposition 3 is proved by the method used in [6, Lemma 5]. This requires a slight modification in the derivation $\mathcal{D}$ associated with the index $\beta$.

Given a real-valued function $f: Y \rightarrow \mathbb{R}, \varepsilon>0$, and a closed subset $P$ of $Y$, define $G(f, \varepsilon, P)$ to be the set of all $y \in P$ such that for every neighborhood $U$ of $y$, there exists $y^{\prime} \in P \cap U$ such that $\left|f(y)-f\left(y^{\prime}\right)\right| \geq \varepsilon$. Let $\mathcal{G}^{0}(f, \varepsilon, P)=P$ and

$$
\mathcal{G}^{1}(f, \varepsilon, P)=\overline{G(f, \varepsilon, P)}
$$

where the closure is taken in $Y$. This defines a derivation $\mathcal{G}$ on the closed subsets of $Y$ which may be iterated in the usual manner. If $\alpha<\omega_{1}$, let

$$
\mathcal{G}^{\alpha+1}(f, \varepsilon, P)=\mathcal{G}^{1}\left(f, \varepsilon, \mathcal{G}^{\alpha}(f, \varepsilon, P)\right) .
$$

If $\alpha<\omega_{1}$ is a limit ordinal, let

$$
\mathcal{G}^{\alpha}(f, \varepsilon, P)=\bigcap_{\alpha^{\prime}<\alpha} \mathcal{G}^{\alpha^{\prime}}(f, \varepsilon, P)
$$

Clearly, the derivation $\mathcal{G}$ is closely related to $\mathcal{D}$. The precise relationship between $\mathcal{D}$ and $\mathcal{G}$ is given in part (c) of the next lemma.

LEMmA 4. If $f$ and $g$ are real-valued functions on $Y, \varepsilon>0$, and $P, Q$ are closed subsets of $Y$, then 
(a) $\mathcal{G}^{1}(f+g, \varepsilon, P) \subseteq \mathcal{G}^{1}(f, \varepsilon / 2, P) \cup \mathcal{G}^{1}(g, \varepsilon / 2, P)$,

(b) $\mathcal{G}^{1}(f, \varepsilon, P \cup Q) \subseteq \mathcal{G}^{1}(f, \varepsilon, P) \cup \mathcal{G}^{1}(f, \varepsilon, Q)$,

(c) $\mathcal{D}^{1}(f, 2 \varepsilon, P) \subseteq \mathcal{G}^{1}(f, \varepsilon, P) \subseteq \mathcal{D}^{1}(f, \varepsilon, P)$.

We leave the simple proofs to the reader. Note that it follows from part (c) that for all $\alpha<\omega_{1}$,

(d) $\mathcal{D}^{\alpha}(f, 2 \varepsilon, P) \subseteq \mathcal{G}^{\alpha}(f, \varepsilon, P) \subseteq \mathcal{D}^{\alpha}(f, \varepsilon, P)$.

Proof of Proposition 3. Parts (a) and (b) of Lemma 4 correspond to $(*)$ and $(* *)$ in $[6$, Lemma 5] respectively. From the proof of that result we obtain, for all $n \in \mathbb{N}$ and $\zeta<\omega_{1}$,

$$
\mathcal{G}^{\omega^{\zeta} \cdot 2 n}(f+g, \varepsilon, Y) \subseteq \mathcal{G}^{\omega^{\zeta} \cdot n}(f, \varepsilon / 2, Y) \cup \mathcal{G}^{\omega^{\zeta} \cdot n}(g, \varepsilon / 2, Y)
$$

Since $\beta_{Y}(f)<\omega^{\xi}$ and $\beta_{Y}(g)<\omega^{\xi}$, there exist $\zeta<\xi$ and $n \in \mathbb{N}$ such that $\beta_{Y}(f)<\omega^{\zeta} \cdot n$ and $\beta_{Y}(g)<\omega^{\zeta} \cdot n$. By $(\mathrm{d})$, for any $\varepsilon>0$,

$$
\mathcal{G}^{\omega^{\zeta} \cdot n}(f, \varepsilon / 2, Y)=\mathcal{G}^{\omega^{\zeta} \cdot n}(g, \varepsilon / 2, Y)=\emptyset
$$

By (d) and (1),

$$
\mathcal{D}^{\omega^{\zeta} \cdot 2 n}(f+g, 2 \varepsilon, Y)=\emptyset .
$$

Since this is true for all $\varepsilon>0$, we have

$$
\beta_{Y}(f+g) \leq \omega^{\zeta} \cdot 2 n<\omega^{\xi} .
$$

TheOREM 5. Let $X$ be a metric space and let $Y$ be an arbitrary subspace of $X$. If $f: Y \rightarrow \mathbb{R}$ satisfies $\beta_{Y}(f)<\omega^{\alpha}$ for some $\alpha<\omega_{1}$, then there exists $F: X \rightarrow \mathbb{R}$ with $\beta_{X}(F) \leq \omega^{\alpha}$ and $F_{\mid Y}=f$.

Proof. Applying Proposition 1 to $f: Y \rightarrow \mathbb{R}$ with $\varepsilon=1 / 2$, we obtain $g_{1}: X \rightarrow \mathbb{R}$ with $\left\|f-g_{1}\right\|_{Y} \leq 1 / 2$ and $\beta_{X}\left(g_{1}\right)<\omega^{\alpha}$. By Proposition 3 we see that $\beta_{Y}\left(f-g_{1}\right)<\omega^{\alpha}$. Now apply Proposition 1 to $\left(f-g_{1}\right)_{\mid Y}$ with $\varepsilon=1 / 2^{2}$. We obtain $g_{2}: X \rightarrow \mathbb{R}$, with $\left\|g_{2}\right\|_{X} \leq\left\|f-g_{1}\right\|_{Y} \leq 1 / 2,\left\|f-g_{1}-g_{2}\right\|_{Y} \leq 1 / 2^{2}$, and $\beta_{X}\left(g_{2}\right)<\omega^{\alpha}$. Continuing in this way, we obtain a sequence $\left(g_{n}\right)$ of real-valued functions on $X$ such that for all $n \in \mathbb{N}$,

(i) $\left\|g_{n+1}\right\|_{X} \leq\left\|f-\sum_{i=1}^{n} g_{i}\right\|_{Y} \leq 1 / 2^{n}$,

(ii) $\beta_{X}\left(g_{n}\right)<\omega^{\alpha}$.

Let $F=\sum_{n=1}^{\infty} g_{n}$. Note that the series converges uniformly on $X$ and $g_{\mid Y}=f$ by (i). Finally, suppose that $\varepsilon>0$. Choose $N$ such that $\sum_{n=N+1}^{\infty}\left\|g_{n}\right\|_{X}$ $<\varepsilon / 4$. Then

$$
\mathcal{D}^{\omega^{\alpha}}(F, \varepsilon, X) \subseteq \mathcal{D}^{\omega^{\alpha}}\left(\sum_{n=1}^{N} g_{n}, \varepsilon / 2, X\right)=\emptyset,
$$

since $\beta_{X}\left(\sum_{n=1}^{N} g_{n}\right)<\omega^{\alpha}$ by Proposition 3. Thus $\beta_{X}(F) \leq \omega^{\alpha}$. 
Corollary 6 (Kuratowski $[7, \S 31, \mathrm{VI}]$ ). Let $X$ be a Polish space and $Y$ be $a G_{\delta}$ subset of $X$. Then every real-valued function of Baire class one on $Y$ can be extended to a function of Baire class one on $X$.

REMARKs. 1. Kuratowski's theorem holds for functions with arbitrary Polish ranges. We do not know if our theorem is true in this more general context.

2. In general, the condition $\beta_{Y}(f)<\omega_{1}$ implies that $f$ is of Baire class one on $Y$, but not vice versa. Indeed, if $Y$ is a subspace of a metric space $X$, then $\beta_{Y}(f)<\omega_{1}$ if and only if $f$ has an extension $f^{\prime}$ to a $G_{\delta}$ subset $Y^{\prime}$ of $X$ such that $\beta_{Y^{\prime}}\left(f^{\prime}\right)=\beta_{Y}(f)$. The two conditions coincide if $Y$ is Polish.

3. Theorem 5 may be viewed as follows: For any $\beta<\omega_{1}$, there exists $\sigma(\beta)<\omega_{1}$ such that if $f$ is a real-valued function defined on a subspace $Y$ of a metric space $X$ with $\beta_{Y}(f)=\beta$, then there exists $F: X \rightarrow \mathbb{R}$ with $\beta_{X}(F) \leq \sigma(\beta)$ and $F_{\mid Y}=f$. (In fact, Theorem 5 shows that if $\beta=\omega^{\alpha}$, then $\sigma(\beta)=\omega^{\alpha+1}$ works.) A natural question is to ask for the optimal (i.e., minimal) value of $\sigma(\beta)$. Theorem 14 and Example 15 together show that $\sigma(1)=3$ is optimal. We do not know the optimal value of $\sigma(\beta)$ for $1<\beta<\omega_{1}$.

2. Extension of continuous functions. In this section, we study the extension of a continuous function on a subspace of a metric space to the whole space. To begin with, we consider the extension of a continuous function from a dense subspace.

Consider a metric space $X$ with a dense subspace $Y$. Suppose that $f$ : $Y \rightarrow \mathbb{R}$ is continuous on $Y$. An obvious way of extending $f$ to $X$ (if $f$ is locally bounded) is to consider the upper limit (or lower limit) of $f$, i.e.,

$$
\widetilde{f}(x)=\limsup _{y \rightarrow x, y \in Y} f(y)=\inf _{\delta>0} \sup _{\substack{(x, y)<\delta \\ y \in Y}} f(y) .
$$

The extended function, which is upper semicontinuous (lower semicontinuous in the case of liminf), is not optimal as far as the oscillation index is concerned. In fact, the limsup extension $\widetilde{f}$ of the continuous function $f$ in Example 15 below has oscillation index $\beta_{X}(\widetilde{f})=\omega$. The following is an alternative algorithm that produces an extension with the smallest possible oscillation index. If $A \subseteq \operatorname{dom} f$, then $\operatorname{osc}(f, A)$ is defined to be $\sup \left\{\left|f(x)-f\left(x^{\prime}\right)\right|: x, x^{\prime} \in A\right\}$. If $x$ belongs to the closure of $\operatorname{dom} f$, then define

$$
\operatorname{osc}(f, x)=\lim _{\delta \rightarrow 0} \operatorname{osc}(f, B(x, \delta) \cap \operatorname{dom} f) .
$$

We first define layers of approximate extensions inductively. Precisely, for each $k \geq 0$, we will choose open sets $S_{k}$ and $X_{k}$ such that $Y \subseteq S_{k} \subseteq X_{k}$, nonnegative integers $\left(n_{k}(s)\right)_{s \in S_{k}}$ and a function $F_{k}: X_{k} \rightarrow \mathbb{R}$. Let $S_{0}=X$ 
and $n_{0}(s)=0$ for all $s \in S_{0}$. Assume that $S_{k}$ has been chosen and $n_{k}(s)$ is defined for all $s \in S_{k}$. Let $\mathcal{U}_{k}=\left\{B\left(s, 2^{-n_{k}(s)}\right): s \in S_{k}\right\}$ and $X_{k}=\bigcup \mathcal{U}_{k}$. Choose a partition of unity $\left(\varphi_{s}^{k}\right)_{s \in S_{k}}$ on $X_{k}$ subordinate to $\mathcal{U}_{k}$. For each $s \in S_{k}$, choose $y_{s}^{k} \in Y \cap B\left(s, 2^{-n_{k}(s)}\right)$. Define $F_{k}: X_{k} \rightarrow \mathbb{R}$ by $F_{k}(x)=$ $\sum_{s \in S_{k}} \varphi_{s}^{k}(x) f\left(y_{s}^{k}\right)$. For each $x \in X_{k}$, let $S_{k}(x)=\left\{s \in S_{k}: x \in \operatorname{supp} \varphi_{s}^{k}\right\}$ and $l_{k}(x)=\max \left\{n_{k}(s): s \in S_{k}(x)\right\}+1$. Note that $S_{k}(x)$ is a finite set since $\left(\operatorname{supp} \varphi_{s}^{k}\right)_{s \in S_{k}}$ is locally finite. Let $S_{k+1}$ be the set of all $x \in X_{k}$ such that $\operatorname{osc}(f, x)<2^{-l_{k}(x)}$. If $x \in S_{k+1}$, choose $n_{k+1}(x) \geq l_{k}(x)$ so that

(1) $\operatorname{osc}\left(f, B\left(x, 2^{1-n_{k+1}(x)}\right) \cap Y\right)<2^{-l_{k}(x)}$,

(2) $B\left(x, 2^{-n_{k+1}(x)}\right) \subseteq B\left(s, 2^{-n_{k}(s)}\right)$ for all $s \in S_{k}(x)$,

(3) $B\left(x, 2^{1-n_{k+1}(x)}\right) \cap \operatorname{supp} \varphi_{s}^{k}=\emptyset$ if $s \in S_{k} \backslash S_{k}(x)$.

The extension $F$ (defined after Lemma 8 ) is obtained by pasting the layers $\left(F_{k}\right)$ one after another. Observe that $X_{k+1} \subseteq X_{k}$ because of condition (2).

Lemma 7. Suppose that $s \in S_{k}, t \in S_{m}$ for some $m>k$, and that $\operatorname{supp} \varphi_{s}^{k} \cap \operatorname{supp} \varphi_{t}^{m} \neq \emptyset$. Then $B\left(t, 2^{-n_{m}(t)}\right) \subseteq B\left(s, 2^{-n_{k}(s)}\right)$.

Proof. Let $x \in \operatorname{supp} \varphi_{s}^{k} \cap \operatorname{supp} \varphi_{t}^{m}$. Then $x \in X_{j}$ for all $j \leq m$. In particular, if $m>j>k$, then there exists $s_{j} \in S_{j}$ such that $x \in \operatorname{supp} \varphi_{s_{j}}^{j}$. Thus it suffices to prove the lemma for $m=k+1$. Assume that $x \in \operatorname{supp} \varphi_{s}^{k} \cap$ $\operatorname{supp} \varphi_{t}^{k+1}$. Note that $s \in S_{k}(t)$. For otherwise, $B\left(t, 2^{1-n_{k+1}(t)}\right) \cap \operatorname{supp} \varphi_{s}^{k}=\emptyset$ by (3). Since $x$ belongs to this set, we have reached a contradiction. It now follows from (2) that $B\left(t, 2^{-n_{k+1}(t)}\right) \subseteq B\left(s, 2^{-n_{k}(s)}\right)$.

Lemma 8. Suppose that $x \in X_{m}$ and $m>k \geq 1$. Then there exists $s \in S_{k}(x)$ such that $\left|F_{k}(x)-F_{m}(x)\right|<2^{1-l_{k-1}(s)}$. Moreover, if $x \in Y$, then $\left|F_{k}(x)-f(x)\right|<2^{-l_{k-1}(s)}$ for some $s \in S_{k}(x)$.

Proof. Denote by $S$ the set of all $t \in S_{m}$ such that $\varphi_{t}^{m}(x)>0$ and choose a point $y \in \bigcap_{t \in S} B\left(t, 2^{-n_{m}(t)}\right) \cap Y$. Let $s$ be an element where $l_{k-1}(s)$ attains its minimum over $S_{k}(x)$. By Lemma $7, B\left(t, 2^{-n_{m}(t)}\right) \subseteq B\left(s, 2^{-n_{k}(s)}\right)$ for all $t \in S$. Hence $\left|f(y)-f\left(y_{t}^{m}\right)\right|<2^{-l_{k-1}(s)}$ for any $t \in S$. By Lemma 7 again, $y \in B\left(t, 2^{-n_{m}(t)}\right) \subseteq B\left(s^{\prime}, 2^{-n_{k}\left(s^{\prime}\right)}\right)$ for all $t \in S$ and all $s^{\prime} \in S_{k}(x)$. Hence

$$
\left|f(y)-f\left(y_{s^{\prime}}^{k}\right)\right|<2^{-l_{k-1}\left(s^{\prime}\right)} \leq 2^{-l_{k-1}(s)}
$$

for all $s^{\prime} \in S_{k}(x)$. Therefore

$$
\begin{aligned}
\left|F_{k}(x)-F_{m}(x)\right| & \leq\left|F_{k}(x)-f(y)\right|+\left|f(y)-F_{m}(x)\right| \\
& <2^{-l_{k-1}(s)}+2^{-l_{k-1}(s)}=2^{1-l_{k-1}(s)} .
\end{aligned}
$$

Moreover, if $x \in Y$, then the above applies for $y=x$. Hence $\left|F_{k}(x)-f(x)\right|<$ $2^{-l_{k-1}(s)}$.

Observe that $l_{k}(s) \geq k+1$ for all $s \in S_{k}, k \geq 0$. It follows from Lemma 8 that $\left(F_{k}\right)$ converges pointwise on $\bigcap X_{k}$ and that the limit is $f$ on $Y$. Define 
$F: X \rightarrow \mathbb{R}$ by

$$
F(x)= \begin{cases}\lim _{k} F_{k}(x) & \text { if } x \in \bigcap X_{k} \\ F_{k}(x) & \text { if } x \in X_{k} \backslash X_{k+1}, k \geq 0 .\end{cases}
$$

Then $F$ is an extension of $f$ to $X$.

Lemma 9. Suppose that $x \in X_{k}$ for some $k \geq 1$. There exists an open neighborhood $U$ of $x$ and $s \in S_{k}(x)$ such that $|F(z)-F(x)|<2^{3-l_{k-1}(s)}$ for all $z \in U$.

Proof. Let $s$ be an element where $l_{k-1}(s)$ attains its minimum over $S_{k}(x)$. Note that $F_{k}$ is continuous on the open set $X_{k}$. Hence there is an open neighborhood $U$ of $x$ such that

(1) $\operatorname{osc}\left(F_{k}, U\right)<2^{-l_{k-1}(s)}$,

(2) $U \subseteq X_{k}$

(3) $U \cap \operatorname{supp} \varphi_{s}^{k}=\emptyset$ if $s \in S_{k} \backslash S_{k}(x)$.

We claim that $S_{k}(z) \subseteq S_{k}(x)$ for all $z \in U$. Indeed, if $z \in U$ and $s \in$ $S_{k}(z) \backslash S_{k}(x)$, then $z \in U \cap \operatorname{supp} \varphi_{s}^{k}=\emptyset$, a contradiction. Now if $z \in U$, then either $z \in X_{m}$ for all $m$ or $z \in X_{m} \backslash X_{m+1}$ for some $m \geq k$. In either case, $\left|F_{k}(z)-F(z)\right| \leq 2^{1-l_{k-1}(s)}$ by Lemma 8 . Therefore,

$$
\begin{aligned}
|F(z)-F(x)| & \leq\left|F(z)-F_{k}(z)\right|+\left|F_{k}(z)-F_{k}(x)\right|+\left|F_{k}(x)-F(x)\right| \\
& <2^{1-l_{k-1}(s)}+2^{-l_{k-1}(s)}+2^{1-l_{k-1}(s)}<2^{3-l_{k-1}(s)} .
\end{aligned}
$$

The next proposition is an immediate consequence of Lemma 9.

Proposition 10. Every $x \in \bigcap X_{k}$ is a point of continuity of $F$.

Proposition 11. If $x \in \mathcal{D}^{1}\left(F, 2^{-m}, X\right) \cap X_{k}, k \geq 1$, then there exists $s \in S_{k}(x)$ such that $l_{k-1}(s) \leq m+3$.

Proof. Since $x \in X_{k}$, by Lemma 9, there exist an open neighborhood $U$ of $x$ and $s \in S_{k}(x)$ such that $|F(z)-F(x)|<2^{3-l_{k-1}(s)}$ for all $z \in U$. Hence $\left|F\left(z_{1}\right)-F\left(z_{2}\right)\right|<2^{4-l_{k-1}(s)}$ for all $z_{1}, z_{2} \in U$. As $x \in \mathcal{D}^{1}\left(F, 2^{-m}, X\right)$, we see that $-m<4-l_{k-1}(s)$. Thus $l_{k-1}(s) \leq m+3$.

Proposition 12. Suppose that $x \in X_{k} \cap \mathcal{D}^{2}\left(F, 2^{-m}, X\right), k \geq 0$. Then $n_{k}(s) \leq m+2$ for all $s \in S_{k}$ such that $\varphi_{s}^{k}(x)>0$.

Proof. Choose an open neighborhood $U_{1}$ of $x$ such that $U_{1} \subseteq\left\{\varphi_{s}^{k}>0\right\}$ for all $s \in S_{k}$ such that $\varphi_{s}^{k}(x)>0$. Note that, in particular, $U_{1} \subseteq X_{k}$. Then choose an open neighborhood $U_{2}$ of $x$ such that $\operatorname{osc}\left(F_{k}, U_{2}\right)<2^{-m}$. Let $U=U_{1} \cap U_{2}$. There exist $z_{1}, z_{2} \in U \cap \mathcal{D}^{1}\left(F, 2^{-m}, X\right)$ such that $\mid F_{k}\left(z_{1}\right)-$ $F_{k}\left(z_{2}\right) \mid \geq 2^{-m}$. If $z_{1}, z_{2} \notin X_{k+1}$, then $F\left(z_{i}\right)=F_{k}\left(z_{i}\right), i=1,2$. This leads to a contradiction with the fact that $\operatorname{osc}\left(F_{k}, U_{2}\right)<2^{-m}$. Thus at least one of $z_{1}, z_{2}$ belongs to $X_{k+1}$. Denote it by $z$. By the previous proposition, there exists $t \in S_{k+1}(z)$ such that $l_{k}(t) \leq m+3$. Let $s \in S_{k}$ be such that $\varphi_{s}^{k}(x)>0$. 
We claim that $s \in S_{k}(t)$. For otherwise, $B\left(t, 2^{1-n_{k+1}(t)}\right) \cap \operatorname{supp} \varphi_{s}^{k}=\emptyset$. This is absurd since the intersection contains the point $z$. It follows from the claim that $l_{k}(t) \geq n_{k}(s)+1$. Hence $n_{k}(s) \leq m+2$, as required.

Proposition 13. $\beta_{X}(F) \leq 3$.

Proof. Suppose that $x \in \mathcal{D}^{3}\left(F, 2^{-m}, X\right)$ for some $m$. Then there exists $k$ such that $x \in X_{k} \backslash X_{k+1}$. Choose a neighborhood $U$ of $x$ such that $U \subseteq B\left(x, 2^{-m-2}\right) \cap X_{k}$ and $\operatorname{osc}\left(F_{k}, U\right)<2^{-m}$. There exist $z_{1}, z_{2} \in$ $U \cap \mathcal{D}^{2}\left(F, 2^{-m}, X\right)$ such that $\left|F\left(z_{1}\right)-F\left(z_{2}\right)\right| \geq 2^{-m}$. If $z_{1}, z_{2} \notin X_{k+1}$, then $F\left(z_{i}\right)=F_{k}\left(z_{i}\right), i=1,2$. This contradicts the fact that $\operatorname{osc}\left(F_{k}, U\right)<2^{-m}$. Hence there exists $z \in U \cap X_{k+1} \cap \mathcal{D}^{2}\left(F, 2^{-m}, X\right)$. By Proposition 12, $n_{k+1}(t) \leq m+2$ for all $t \in S_{k+1}$ such that $\varphi_{t}^{k+1}(z)>0$. Fix such a $t$. Note that

$$
d(x, t) \leq d(x, z)+d(z, t)<2^{-m-2}+2^{-n_{k+1}(t)} \leq 2^{1-n_{k+1}(t)} .
$$

Thus

$$
\operatorname{osc}(f, x) \leq \operatorname{osc}\left(f, B\left(t, 2^{1-n_{k+1}(t)}\right) \cap Y\right)<2^{-l_{k}(t)} .
$$

We claim that $S_{k}(x) \subseteq S_{k}(t)$. For otherwise, there exists $s \in S_{k}(x) \backslash S_{k}(t)$. Then $B\left(t, 2^{1-n_{k+1}(t)}\right) \cap \operatorname{supp} \varphi_{s}^{k}=\emptyset$. This is absurd since the intersection contains the point $x$. It follows from the claim that $l_{k}(t) \geq l_{k}(x)$. Hence $\operatorname{osc}(f, x)<2^{-l_{k}(x)}$. Then $x \in S_{k+1} \subseteq X_{k+1}$, a contradiction.

Theorem 14. Let $X$ be a metric space and $Y$ be a subspace of $X$. Every continuous function $f$ on $Y$ can be extended to a function $F$ on $X$ with $\beta_{X}(F) \leq 3$.

Proof. Applying the preceding lemmas and propositions, we obtain an extension $\widetilde{f}$ of $f$ to $\bar{Y}$ such that $\beta_{\bar{Y}}(\widetilde{f}) \leq 3$. By [8, Theorem 3.6], there is a further extension $F$ of $\tilde{f}$ to $X$ such that $\beta_{X}(F)=\beta_{\bar{Y}}(\widetilde{f}) \leq 3$. (Note that the proof of [8, Theorem 3.6] does not require the compactness of $X$.)

The following example shows that Theorem 14 is optimal.

EXAMPLE 15. There is a subspace $Y \subseteq\{0,1\}^{\omega}=X$ and a continuous real-valued function $f$ on $Y$ such that $\beta_{X}(F) \geq 3$ for any extension $F$ of $f$ to $X$.

Proof. For any integer $n$, denote $n(\bmod 2)$ by $\widehat{n}$. Let

$$
Y=\left\{\left(\varepsilon_{i}\right) \in X: \varepsilon_{i}=0 \text { for infinitely many } i \text { 's }\right\} .
$$

We denote elements in $X$ of the form

$$
(\underbrace{1, \ldots, 1}_{n_{1}}, 0, \underbrace{1, \ldots, 1}_{n_{2}}, 0, \ldots, \underbrace{1, \ldots, 1}_{n_{k}}, 0, \ldots)
$$


by $\left(1^{n_{1}}, 0,1^{n_{2}}, 0, \ldots, 1^{n_{k}}, 0, \ldots\right)$. Also write $\left(\varepsilon_{1}, \ldots, \varepsilon_{k}, \varepsilon, \varepsilon, \ldots\right)$ as $\left(\varepsilon_{1}, \ldots\right.$, $\left.\varepsilon_{k}, \varepsilon^{\omega}\right), \varepsilon_{i}, \varepsilon \in\{0,1\}$. Define $g: Y \rightarrow X$ by

$$
g\left(1^{n_{1}}, 0,1^{n_{2}}, 0, \ldots, 1^{n_{k}}, 0, \ldots\right)=\left(\widehat{n}_{1}, \widehat{n}_{2}, \ldots\right), \quad n_{1}, n_{2}, \cdots \in \mathbb{N} \cup\{0\},
$$

and let $h: X \rightarrow \mathbb{R}$ be the canonical embedding of $X$ into $\mathbb{R}, h\left(\varepsilon_{1}, \varepsilon_{2}, \ldots\right)=$ $\sum_{k=1}^{\infty} 2 \varepsilon_{k} / 3^{k}$. Then the function $f=h \circ g: Y \rightarrow \mathbb{R}$ is continuous. Suppose that $F$ is an extension of $f$ to $X$ such that $\beta_{X}(F) \leq 2$. First observe that for any $n_{1}, \ldots, n_{k} \in \mathbb{N} \cup\{0\}$ and all $n \in \mathbb{N}$,

$\left|F\left(1^{n_{1}}, 0, \ldots, 1^{n_{k}}, 0,1^{2 n}, 0^{\omega}\right)-F\left(1^{n_{1}}, 0, \ldots, 1^{n_{k}}, 0,1^{2 n-1}, 0,1,0,1, \ldots\right)\right|=\frac{1}{3^{k}}$.

Hence $\left(1^{n_{1}}, 0, \ldots, 1^{n_{k}}, 0,1^{\omega}\right) \in \mathcal{D}^{1}\left(F, 1 / 3^{k}, X\right)$. Let $F\left(1^{\omega}\right)=a$. Either $|a| \geq$ $1 / 2$ or $|1-a| \geq 1 / 2$. We assume the former; the proof for the latter case is similar. Since $\left(1^{\omega}\right) \notin \mathcal{D}^{2}(F, 1 / 3, X)$, there exists a neighborhood $U$ of $\left(1^{\omega}\right)$ such that $|F(x)-a|<1 / 3$ if $x \in U \cap \mathcal{D}^{1}(F, 1 / 3, X)$. In particular, there exists $n_{1} \in \mathbb{N}$ such that

$$
\left|F\left(1^{2 n_{1}}, 0,1^{\omega}\right)-a\right|=\frac{1}{3}-\delta \quad \text { for some } \delta>0 .
$$

Similarly, using the fact that $\left(1^{2 n_{1}}, 0,1^{\omega}\right) \notin \mathcal{D}^{2}\left(F, 1 / 3^{2}, X\right)$, we obtain some $n_{2} \in \mathbb{N}$ such that

$$
\left|F\left(1^{2 n_{1}}, 0,1^{2 n_{2}}, 0,1^{\omega}\right)-F\left(1^{2 n_{1}}, 0,1^{\omega}\right)\right|<\frac{1}{3^{2}} .
$$

Continuing, we choose $n_{1}, n_{2}, \ldots \in \mathbb{N}$ such that

$$
\left|F\left(1^{2 n_{1}}, 0, \ldots, 1^{2 n_{k+1}}, 0,1^{\omega}\right)-F\left(1^{2 n_{1}}, 0, \ldots, 1^{2 n_{k}}, 0,1^{\omega}\right)\right|<\frac{1}{3^{k+1}}, \quad k \in \mathbb{N} .
$$

In particular,

$$
\left|F\left(1^{2 n_{1}}, 0, \ldots, 1^{2 n_{k}}, 0,1^{\omega}\right)-a\right| \leq \frac{1}{3}+\frac{1}{3^{2}}+\cdots-\delta=\frac{1}{2}-\delta, \quad k \in \mathbb{N} .
$$

Since $|a| \geq 1 / 2$, we have $\left|F\left(1^{2 n_{1}}, 0, \ldots, 1^{2 n_{k}}, 0,1^{\omega}\right)\right| \geq \delta$ for all $k \in \mathbb{N}$. But

$$
F\left(1^{2 n_{1}}, 0, \ldots, 1^{2 n_{k}}, 0,1^{2 n}, 0^{\omega}\right)=f\left(1^{2 n_{1}}, 0, \ldots, 1^{2 n_{k}}, 0,1^{2 n}, 0^{\omega}\right)=0
$$

for all $n \in \mathbb{N}$. Hence $\left(1^{2 n_{1}}, 0, \ldots, 1^{2 n_{k}}, 0,1^{\omega}\right) \in \mathcal{D}^{1}(F, \delta, X)$ for all $k \in \mathbb{N}$. However, note that the sequence $\left(\left(1^{2 n_{1}}, 0, \ldots, 1^{2 n_{k}}, 0,1^{\omega}\right)\right)_{k \in \mathbb{N}}$ converges to the point $\left(1^{2 n_{1}}, 0, \ldots, 1^{2 n_{j}}, 0,1^{2 n_{j+1}}, 0, \ldots\right)$ and

$$
\begin{aligned}
& \left|F\left(1^{2 n_{1}}, 0, \ldots, 1^{2 n_{k}}, 0,1^{\omega}\right)-F\left(1^{2 n_{1}}, 0, \ldots, 1^{2 n_{j}}, 0,1^{2 n_{j+1}}, 0, \ldots\right)\right| \\
& \quad=\left|F\left(1^{2 n_{1}}, 0, \ldots, 1^{2 n_{k}}, 0,1^{\omega}\right)-f\left(1^{2 n_{1}}, 0, \ldots, 1^{2 n_{j}}, 0,1^{2 n_{j+1}}, 0, \ldots\right)\right| \\
& \quad=\left|F\left(1^{2 n_{1}}, 0, \ldots, 1^{2 n_{k}}, 0,1^{\omega}\right)\right| \geq \delta
\end{aligned}
$$

for all $n \in \mathbb{N}$. Therefore, $\left(1^{2 n_{1}}, 0, \ldots, 1^{2 n_{j}}, 0,1^{2 n_{j+1}}, 0, \ldots\right) \in \mathcal{D}^{2}(F, \delta, X)$, contrary to the assumption that $\beta_{X}(F) \leq 2$. 
REMARK. With regard to the question raised in Remark 3 of $\S 1$, we have been able to show that if $Y$ is a subspace of a countable ordinal $X$ (not necessarily compact), and $f: Y \rightarrow \mathbb{R}$ satisfies $\beta_{Y}(f) \leq 3$, then there is an extension $F: X \rightarrow \mathbb{R}$ of $f$ such that $\beta_{X}(F) \leq \beta_{Y}(f)+1$.

\section{References}

[1] R. L. Blair, Extensions of continuous functions from dense subspaces, Proc. Amer. Math. Soc. 54 (1976), 355-359.

[2] A. M. Bruckner, J. B. Bruckner and B. S. Thomson, Real Analysis, Prentice-Hall, Upper Saddle River, NJ, 1997.

[3] J. Dugundji, Topology, Allyn and Bacon, Boston, 1966.

[4] S. Hernández, Extension of continuous functions into uniform spaces, Proc. Amer. Math. Soc. 97 (1986), 355-360.

[5] O. Kalenda and J. Spurný, Extending Baire-one functions on topological spaces, Topology Appl. 149 (2005), 195-216.

[6] A. S. Kechris and A. Louveau, A classification of Baire class 1 functions, Trans. Amer. Math. Soc. 318 (1990), 209-236.

[7] C. Kuratowski, Topologie, Vol. I, 4ème éd., Monograf. Mat. 20, PWN, Warszawa, 1958.

[8] D. H. Leung and W.-K. Tang, Functions of Baire class one, Fund. Math. 179 (2003), $225-247$.

D. H. Leung

Department of Mathematics

National University of Singapore

2 Science Drive 2

Singapore 117543

E-mail: matlhh@nus.edu.sg
W.-K. Tang Mathematics and Mathematics Education National Institute of Education Nanyang Technological University 1 Nanyang Walk Singapore 637616 E-mail: wktang@nie.edu.sg

Received 27 February 2006;

in revised form 18 July 2006 